\title{
Design of All Digital Flight Program Training Desktop Application System
}

\author{
Yu Li ${ }^{1, a}$, Gang $\mathrm{An}^{2, \mathrm{~b}}$, Xin $\mathrm{Li}^{3, \mathrm{c}}$ \\ ${ }^{1}$ System Integration Center, FAI AVIC,Xi'an, CN \\ ${ }^{2}$ School of management, Xi'an Jiaotong university, Xi'an, CN \\ ${ }^{3}$ Landing Gear System Research Institute, FAI AVIC, Xi'an, CN
}

\begin{abstract}
All digital flight program training desktop application system operating conditions are simple. Can make the aircraft aircrew learning theory and operation training closely. Improve the training efficiency and effectiveness. This paper studies the application field and design requirements of flight program training system. Based on the WINDOWS operating system desktop application, the design idea and system architecture of the all digital flight program training system are put forward. Flight characteristics, key airborne systems and aircraft cockpit are simulated. Finally, By comparing flight training simulator and the specific script program training system, The characteristics and advantages of the training system are analyzed in this paper.
\end{abstract}

\section{Introduction}

Modern aircraft set a variety of complex systems and high-tech in one. The function and performance improvement are also used to aircrew put forward higher requirements. The traditional training mode has many problems, mainly reflected in: backward technology, single method, scattered resources, sharing difficulties, training is not flexible, intuitive, systematic and operability, low degree of automation, the lack of effective supervision and evaluation, and to deal with the new situation of the training needs. With the rapid development of computer, network, multimedia, digital simulation and modeling technology, by means of information to compensate for the lack of traditional training mode, carry out the resources development, management and training, improve the training quality and efficiency, has become the inevitable trend of development of modern flight training[1].

All digital flight program training desktop application system running on the WINDOWS OS, the minimum operating and training units for a single desktop computer or portable PC. And can flexibly set up the training room through the high speed Ethernet environment. Support single machine training mode, double pilot collaborative training mode, distributed synchronization teaching mode. For the aircrew learning theory, cognition, cockpit equipment operating procedures and flight training, maintenance training etc.

\section{System architecture design}

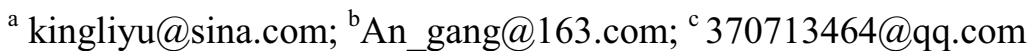




\subsection{Functional composition}

The function of the all digital flight program training desktop application system is shown in figure 1 .

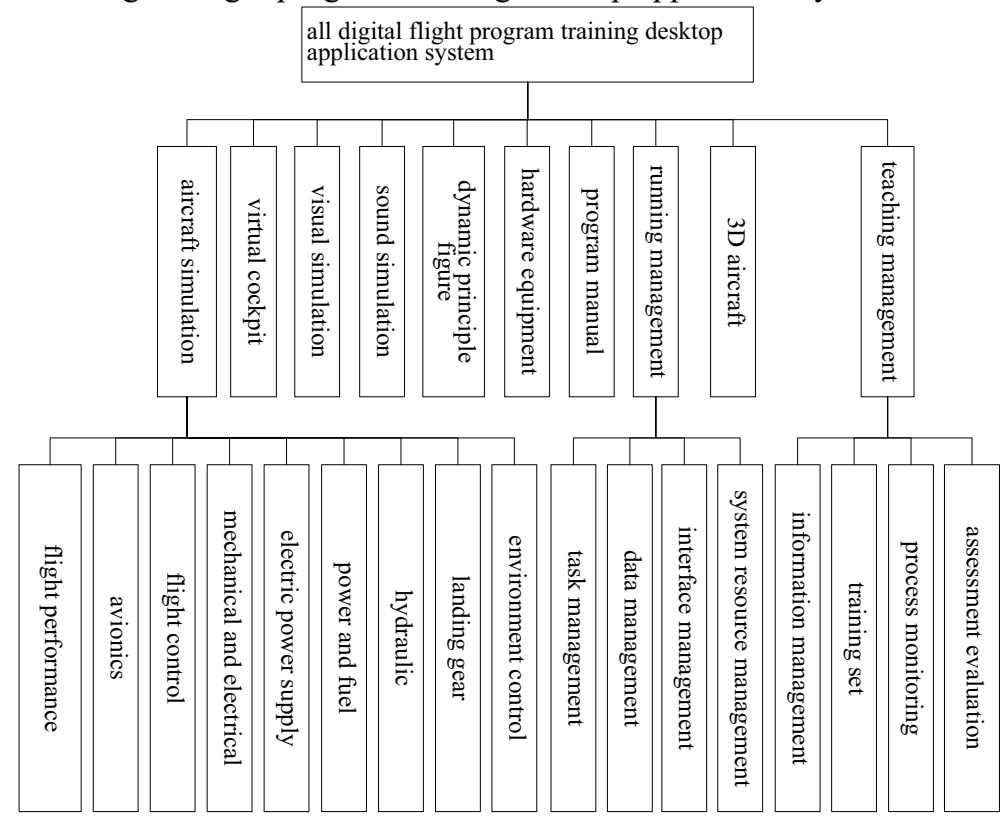

Figure 1. Functional composition

Aircraft simulation: including flight simulation and airborne system simulation. Mainly completes the flight performance simulation. Functional simulation of airborne system, working logic simulation and Simulation of cross link between systems[2].

Virtual cockpit: Construction of the 3D virtual cockpit environment consistent with a real aircraft. For the cockpit and cabin equipment cognition, aircrew flight operation training, emergency procedure training, power checking operation on ground, operation testing etc..

Visual simulation: the use of computer image generation technology, produce a realistic external scene, the simulation of the airport, route and flight base, including the corresponding airport runway, route terrain and terrain, etc..

Sound simulation: the simulation of the aircraft during the flight can be heard in a variety of sounds, including a variety of environmental noise, equipment noise, system tips and alarm voice, etc..

Dynamic principle figure: The simulation of the airborne system principle. The cross-linking relationship simulation of the different airborne systems. For the crew to learn and master the working process of the system.

Hardware equipment: the smallest unit of software running for a single desktop computer or portable PC, and can be through the server, high speed Ethernet to achieve virtual classroom or multi machine network training.

Running management: system scheduling, management and operation control, including task management, data management, interface management and system resource management.

Program manual: the training management system to load the aircraft's flight class and maintenance related to the electronic technical manuals, and the current program associated with the system training.

3D aircraft: The 3D aircraft environment that is consistent with a real aircraft, including the fuselage, wing, cargo hold, floor, and system layout. Through the training management system scheduling, and the simulation can be synchronized with the program, the implementation of different training operating procedures. 
Teaching management: information management, training seting and process monitoring, assessment evaluation etc. To achieve the purpose of training class setting, system initialization settings, a variety of flight parameters, airport conditions, the status of the aircraft display and settings, aircraft failure and special circumstances set, flight training process monitoring, etc..

\subsection{Software architecture}

The all digital flight training program desktop application system based on operation management system as the core, the integrated simulation system, virtual cockpit system, visual simulation, sound simulation, dynamic principle and teaching management system, realize the whole system software and hardware resource management, data exchange, task scheduling and management. The system architecture is shown in figure 2. The internal function of the system is highly cohesive and loosely coupled, and the system can transfer and exchange the data flow and control flow through the common data pool, so as to achieve the flexible resolution and assembly of the software[3].

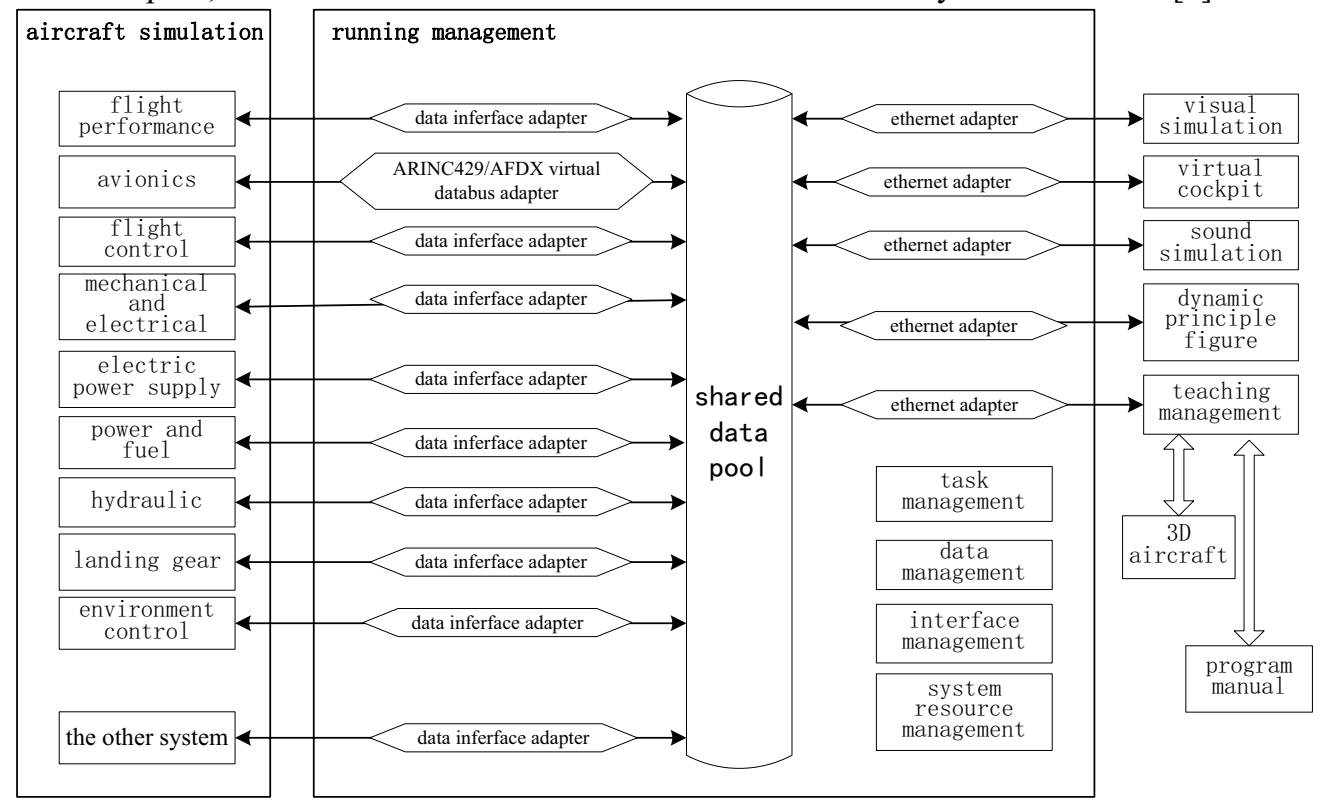

Figure 2. Software architecture

Operation management system mainly includes: public data pool, is responsible for the data exchange between the system; task management, task scheduling, task group is responsible for the rate allocation and management, process / thread allocation and management; data management, responsible for public data pool establishment, data update management, data read / write operation management interface; the management of the data exchange interface conversion, computer network communication interface for each subsystem and the public data pool management system; resource management, management of the operating system software resources and computer hardware resources such as CPU, memory, hardware interface etc.. Run management system to meet the WINDOWS desktop application system task management, resource management, interface management, data management, software architecture, network architecture, etc..

The cross linking of the avionics system simulation and operation management system. Data exchange between public data pool and the avionics system through ARINC429 and AFDX virtual 
bus data adapter software. visual simulation, virtual cockpit, dynamic principle, training management and operation management system linking, through the Ethernet interface adapter software and the public data pool data exchange; conversion flight simulation and flight control system simulation, mechanical and electrical system simulation, power system simulation, power/fuel system simulation, hydraulic system simulation, simulation, gear ring, door control simulation simulation system through the software interface adapter to exchange data and the public data pool. The 3D plane and procedure manuals and training management system directly connected by Ethernet, the main transmission training information and training process control instructions and procedures manual information to realize the training process of synchronous scheduling and operation of related subjects and the corresponding three-dimensional plane information.

\subsection{Network teaching}

The virtual training classroom can be flexibly set up through the high speed Ethernet environment, as shown in figure 3 . The virtual training room is composed of one system server, one teacher computer, one training administrator computer, one system administrator computer and several student training computers.

The training computer's user interface is formed by the 4 display of the computer, and the display content can be independently arranged on each screen. The commonly used display mode in the training process:

- No. 1 display: attitude, participation, representation of a page.

- No. 2 display: Avionics navigation information.

- No. 3 display: by operating the dynamic way roaming the entire virtual cockpit.

- No. 4 display: you can choose to display the training manual, dynamic principle diagram, three-dimensional plane etc..

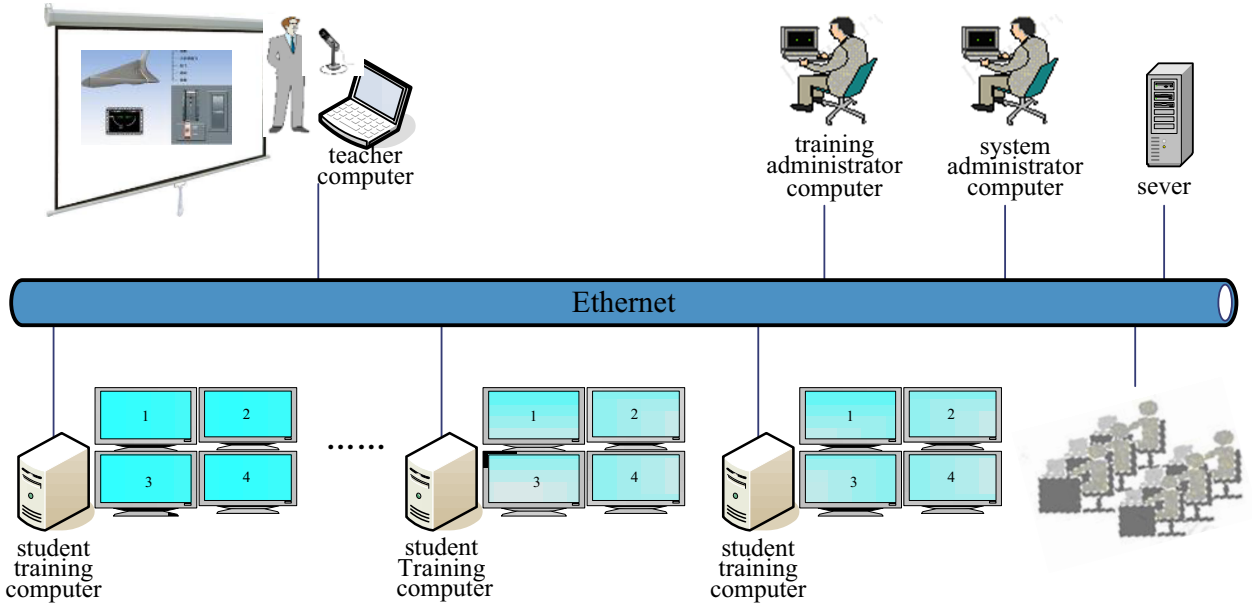

Figure 3. Virtual training classroom

\section{System simulation design}

\subsection{Aircraft simulation modeling}

The principle of aircraft simulation is shown in figure 4. Flight simulation includes: Aerodynamic module, mass features module, atmospheric environment module, wind module, turbulence module, shear module, reposition module, ground control calculation module and equation of motion[4]. Airborne system simulation includes[5]: 
- Function simulation modeling of airborne system.

- Simulation of the interaction between cross linked systems.

- Simulation cockpit control panel for the control of the system and control box lights.

- Simulate common fault, alarm and display.

- Simulation of flight inspection procedures and display control.

\subsection{Virtual cockpit simulation}

Cockpit simulation is one of the most important systems simulation of the whole digital flight program, Requirements for the simulation of particle size to reach the component level. The effective area of the system simulation of the entire cockpit to ensure real aircraft cockpit simulation reappearance. Simulation of all the control and display equipment in the cockpit through modeling method[6]. A variety of display, indicator, instrument, switch, control box, switch, handle, button, indicator, knob, adjustment device, operation instructions and response in line with the actual aircraft. Shape, size, color, brightness, etc. with the real aircraft. Through the cabin glass to watch the scene outside the cabin, through the mouse and keyboard operation on the virtual cockpit to promote / zoom / translation / rotation and other roaming operations.

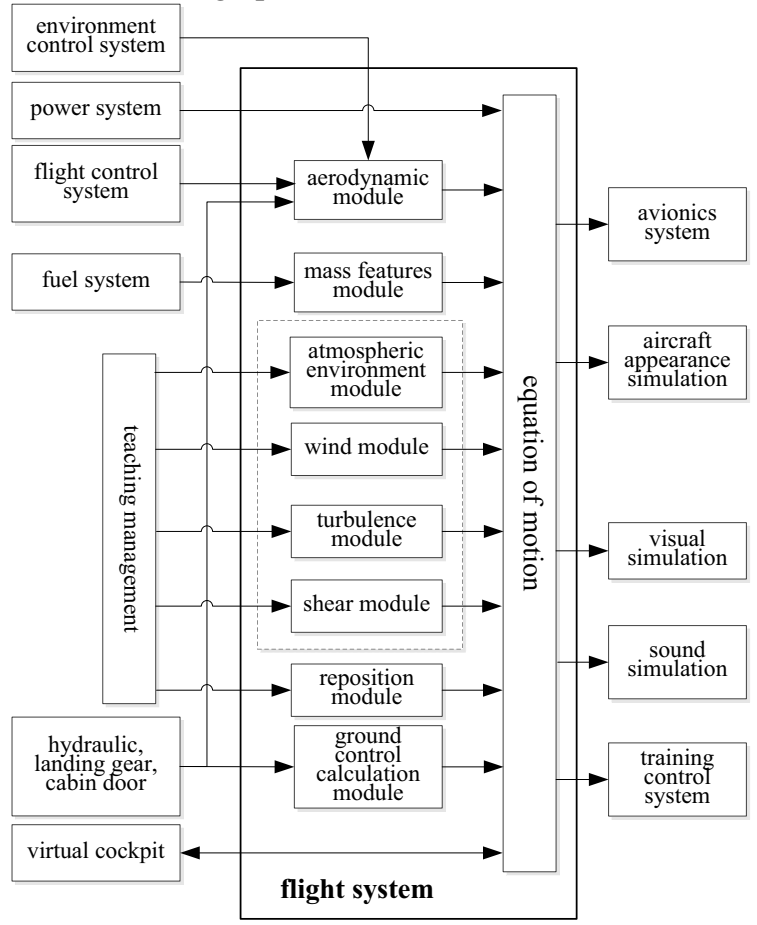

Figure 4. Aircraft simulation principle

According to the characteristics of "WINDOWS OS + desktop computer" with keyboard and mouse as the main input device, the cockpit operating mode (such as: rod, wheel, pedal, button, pulling the handle, with a protective cover switch, a trackball, keyboard and so on), to optimize the design of different equipment and operating characteristics, through the realization of common the keyboard and mouse to complete the cockpit all operations, and meet the design requirements of reasonable operation, intuitive and simple. 
Trajectory ball operation switching model optimization: through the virtual cockpit software to re development of the avionics system, the trajectory of the ball function, so that the original independent avionics software is fully integrated into the virtual cockpit. As long as the students in the virtual cockpit view through the "CTRL + mouse" can make the Windows ordinary mouse operation fast and flexible switch for the control system of the track ball operation.

The throttle trim, handle operation optimization: throttle four engines can be operated at the same time, also can operate independently, through simple mouse control is difficult, therefore, the navigation window set in virtual cockpit in the auxiliary control work area, add a slide bar to achieve four engines control.

\subsection{Teaching management design}

The teaching management system for teaching and training of the whole process of information management, training set, process monitoring and assessment evaluation etc..

Information management functions, including:

- Teachers, students access to login and basic information management.

- The system can automatically establish and maintain the teaching and training of teachers and students, the examination of the file.

- The system supports the basic information of the teaching staff, training information and assessment results of the query.

- Record system status information, such as: technical state, system version change, etc..

Training set function, including:

- Course setting.

- System parameter setting.

- Aircraft initialization setting.

- Flight environment initialization setting.

- System fault setting.

Process monitoring functions, including:

- System operation control, such as start/restart, pause, stop.

- Automatically record the student assessment process, including the status of the aircraft system, operating procedures, the location of snapshots and simulation operation and other information.

- The teacher management student assessment authority, monitoring students' training and evaluation process.

- $\quad$ The teacher to maintain management system.

The execution of the whole system is completed by the user, the training computer and the server. The system operation process mainly includes: the server starts, the virtual training system start, the edition automatic renewal, the user registers, the teaching / training pattern establishment, the training and the movement control[7]. The timing logic of the operation is shown in figure 5. 


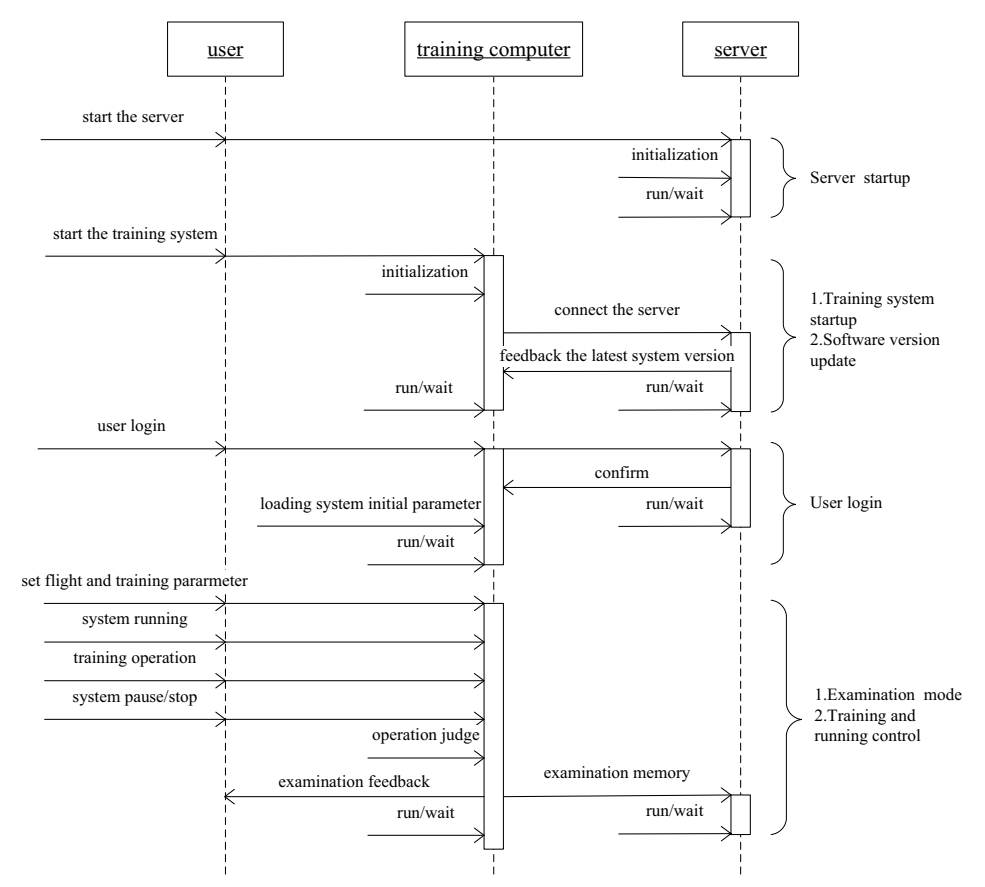

Figure 5. Operational process sequence logic

\subsection{Teaching mode design}

The design of teaching mode in high speed Ethernet environment is shown in Figure 6.

Single machine training mode consists of a single training computer. Students can be on the computer virtual instructors guide training, autonomous training and evaluation.

Double pilot collaborative training mode from two sets of training computer composed of a complete set of two driving units. Students can be on the computer virtual instructors guide training, autonomous training and evaluation.

Distributed synchronization teaching mode, one computer training as a teacher teaching, student learning as other computer systems, to provide different permissions for identification of the teacher when the flight operations performed in synchronous computer students. 


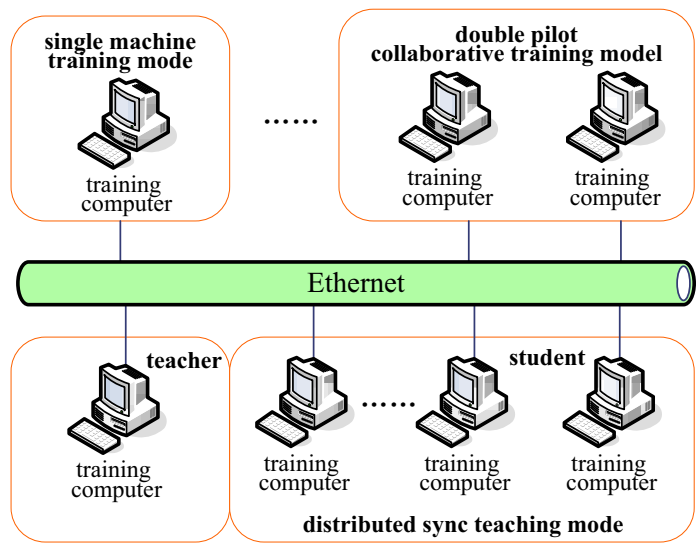

Figure 6. Teaching mode

\section{Conclusions}

The comparison of the all digital flight program training desktop application system and the flight training simulator: The flight training simulator is more suitable for the training of the pilot's flying skills, it is the advanced training based on the solid theoretical foundation and skilled operating skills. Due to the high cost of manufacturing, use and maintenance, limiting the number of equipment and training time. Flexibility difference is difficult to meet the requirements of autonomous learning and differentiated training.

The all digital flight training program desktop application system suitable for aircrew learning theory, cognitive equipment and flight operations training program. The system runs in WINDOWS OS, supports single machine or network teaching and training, the hardware resources and the use condition request is simple. The system can meet the training of self training, classroom teaching, automatic evaluation and other aspects of the system, and the training cost is low. All digital flight simulation technology and simulation software can be easily and efficiently transplanted or reused to the aircraft simulation platform, simulator, virtual reality training system, which has a good value for popularization and application.

\section{References}

[1] Huang Zhongrong, Liang Yunduan. Flight training system and development of trainer. training plane, 2011.

[2] Fang Zhenping, Chen Wanchun, Zhang Shuguang. Aircraft flight mechanics. Beijing: Beihang University press, 2012.

[3] Zhi Chaoyou, Li Zhenshui, Xue Feng. Research on virtual test system framework based on model component. Computer measurement and control, 2011.

[4] Jia Qiuling, Yuan Dongli. Simulation, analysis and design based on MATLAB7.X/Simulink/Stateflow system. Northwestern Polytechnical University press, 2008.

[5] Wang Yong, Yu Hongkun. Airborne computer system. Beijing: National Defense Industry Press. 2008.

[6] Yao Xudong. Design and implementation of avionics system simulation platform based on virtual instrument. Shanghai Jiao Tong University, 2012.

[7] Zhi Chaoyou, Tang Changhong. Development of modern aircraft system virtual test verification technology. Aviation science and technology, 2010. 